\title{
Berberine Inhibits Cell Proliferation by Interfering with Wild-Type and Mutant P53 in Human Glioma Cells
}

This article was published in the following Dove Press journal: OncoTargets and Therapy

\author{
Ziqiang Liu (D) \\ Yong Chen' \\ Haijun Gao (D) \\ Weidong Xu (D) \\ Chaochao Zhang' \\ Jiacheng Lai ${ }^{1}$ \\ Xingxing $\mathrm{Liu}^{2}$ \\ Yuxue Sun ${ }^{3}$ \\ Haiyan Huang'
}

'Department of Neurosurgery, The First Hospital of Jilin University, Changchun, People's Republic of China; ${ }^{2}$ Department of Internal Medicine, The First Hospital of Jilin University, Changchun, People's Republic of China; ${ }^{3}$ Department of Neurosurgery, Henan Provincial People's Hospital, Zhengzhou, People's Republic of China
Correspondence: Haiyan Huang; Yuxue Sun

Department of Neurosurgery, The First Hospital of Jilin University, Changchun, People's Republic of China

Tel +86-I3578967878; +86-16638060663

Email huanghy@jlu.edu.cn;

doctoryuxue@hotmail.com
Introduction: Glioma is the most common malignant brain tumor. TP53 is the most common mutant gene in human cancer. Wild-type p53 (wtp53) is a tumor suppressor protein whereas mutant p53 (mutp53) is an oncoprotein that promotes tumor cell proliferation. Our aim was to examine the inhibitory effects of berberine on the proliferation of human glioma cells via regulation of wtp53, mutp53, and their downstream molecules.

Methods: We selected wtp53 cells (U87 cells) and mutp53 cells (U251 cells termed p53 $\mathrm{R} 273 \mathrm{H})$ to examine the inhibitory effects of berberine on human glioma cells. We used the CCK-8 kit to detect the toxic effect of berberine. Flow cytometry was used to detect the effect of berberine. Clone formation test was used to test the inhibitory effect of berberine on the proliferation of glioma cells. Western blot was used to detect the changes of related proteins such as p53, p-p53, p21 and cyclin D1. Lentivirus transduction was used to transduce wild-type p53 into U251 cells to further examine the effect of berberine. The nude mouse subcutaneous tumor model was used to detect the effect of berberine on inhibiting the proliferation of glioma cells in vivo.

Results: Berberine promoted the phosphorylation of wtp53, increased the expression of p21 protein, reduced cyclin D1 content, and caused G1 phase arrest in U87 cells. Berberine also reduced mutp53 content and caused G2 phase arrest in U251 cells with a concurrent decrease in p21, cyclin D1, and cyclin B1 content. Transduction with wtp53 enhanced the effects on cell cycle arrest. Further, berberine significantly inhibited glioma growth in vivo mouse tumor model. Discussion: Glioma is a group of heterogeneous brain tumors with unique biological and clinical characteristics. Berberine can inhibit glioma cells through a variety of ways. Our research indicated that berberine inhibited the proliferation of glioma cells by interfering with wtp53 and mutp53. This indicates that berberine could be used as a potential drug to treat wild-type and mutant p53 glioma.

Keywords: berberine, wild-type p53, mutant p53, cell proliferation, glioma

\section{Introduction}

Glioma is the most common primary tumor in the central nervous system, accounting for approximately $81 \%$ of intracranial malignancies. ${ }^{1}$ It is characterized by a variety of somatic mutations and abnormal activation of inflammatory reactions. ${ }^{2}$ Of the gliomas, glioblastoma has the poorest prognosis, ${ }^{3}$ with a median survival time of $14-17$ months and a 5-year survival rate of only $5 \%{ }^{4}$ For malignant gliomas, postoperative temozolomide (TMZ) combined with chemotherapy is typically used as standard treatment, ${ }^{5}$ but treatment results remain suboptimal. 
p53 is a well-established tumor suppressor protein. In stress conditions such as DNA damage or hypoxia, p53 is activated to participate in DNA repair and cell cycle regulation, differentiation, metabolism, autophagy, apoptosis, ${ }^{6-8}$ and inhibition of tumor cell proliferation and invasion. ${ }^{9}$ Missense mutations in the TP53 gene result in loss-offunction of wild-type p53 (wtp53), and mutant p53 (mutp53) negatively regulates the remaining wtp53. ${ }^{10,11}$ mutp53 also has carcinogenic functions that are entirely independent of wtp53, and acts as a cancer factor that promotes tumor cell proliferation, inhibits apoptosis, and produces drug resistance. ${ }^{12}$ Under classical conditions, wtp53 induces the expression of p21 and cyclin D1. p21 is a strong inhibitor of cyclin $\mathrm{D} 1$ protein, which regulates the cell cycle process and controls cell proliferation or quiescence. ${ }^{13}$ Studies have indicated that mutp53 can still induce p21 and cell cycle arrest. ${ }^{14-16}$ Nevertheless, the role of mutp53 in the cell cycle remains to be explored.
Berberine (BBR, Figure 1A) is an isoquinoline alkaloid which is a natural compound in traditional Chinese medicine and can be isolated from many Chinese herbal medicines. Berberine has anti-inflammatory, anti-microbial, hypoglycemic, and lipid-lowering pharmacological effects. ${ }^{17,18}$ In addition, berberine has been shown to have anti-cancer properties and can suppress the growth of cancer cells in gastric, bladder, colon, glioma, breast, melanoma, pancreatic, endometrial, and lung cancers. ${ }^{19-21}$ Studies have demonstrated that berberine exerts anti-cancer effects by regulating MAPK, Erk1/2, JNK, AKT, and Wnt/ $\beta$-catenin signaling pathways. ${ }^{20,22}$ However, the role of berberine in signaling pathways downstream of wtp53 and mutp53 is unclear.

In this study, we selected wtp53 cells (U87 cells) and mutp53 cells (U251 cells termed p53 R273H) to examine the inhibitory effects of berberine on human glioma cells. The results suggested that mutp53 might directly promote the transcription of cyclin D1 protein, and berberine could
A<smiles>COc1ccc2cc3[n+](cc2c1OC)CCc1cc2c(cc1-3)OCO2</smiles>

Berberine chloride
B

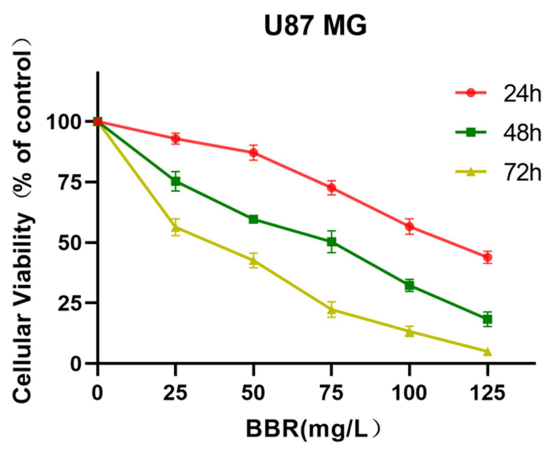

C

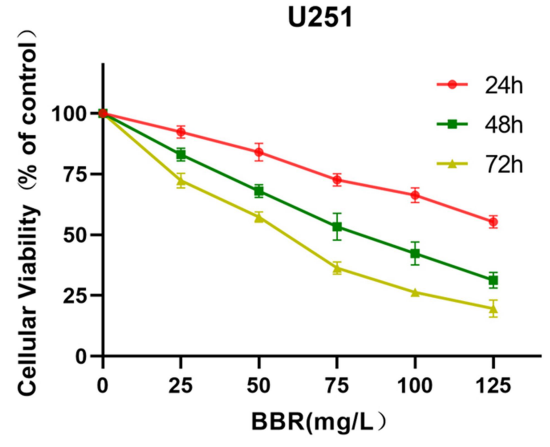

D

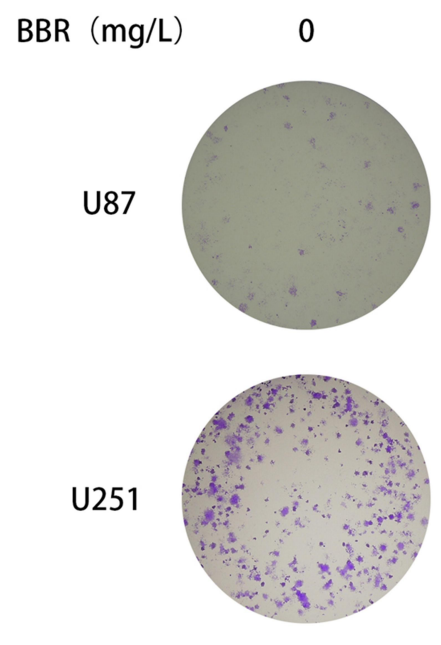

1.25
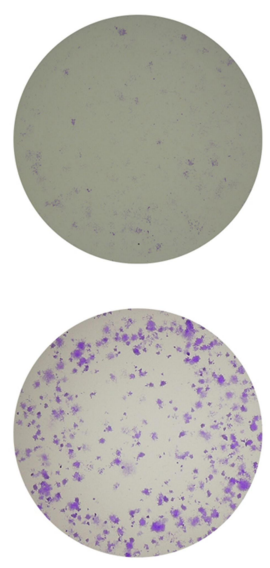

2.5
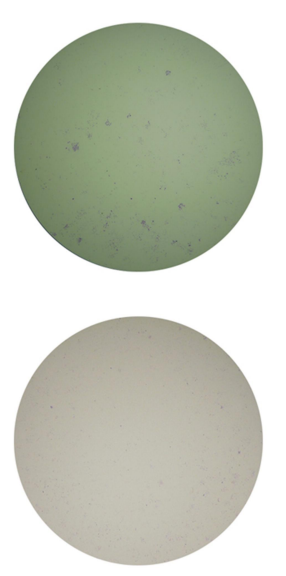

5
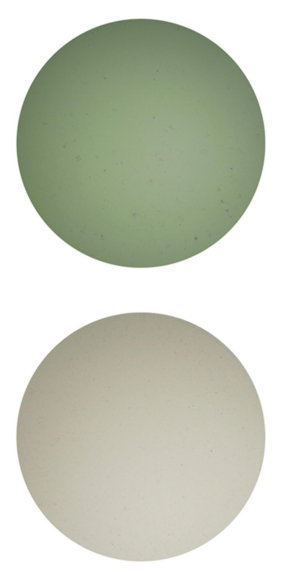

Figure I Berberine inhibited cell growth and reduced cell proliferation in glioma cells. (A) Structural formula of berberine hydrochloride. (B and C) CCK-8 detected toxic effects of berberine at different concentrations on U87 and U25I cells for 24,48 , and 72 hours. (D) The clone formation experiment showed the effect of berberine on the proliferation ability of U87 and U25I cells. With the increase of berberine concentration, the proliferation ability of glioma cells decreased gradually. 
not only promote the phosphorylation of wtp53, but also accelerate the degradation of mutp53, thus causing cell cycle arrest.

\section{Materials and Methods}

\section{Reagents}

Berberine was purchased from Chroma Biotechnology Company (Chengdu, China) with a purity of up to $98 \%$. Berberine was dissolved in dimethyl sulfoxide (DMSO) and subsequently diluted to the required concentrations with complete cell culture medium, with a final DMSO concentration of $<0.1 \%$. Dulbecco's modified Eagle's medium (DMEM), fetal bovine serum (FBS) were purchased from Thermo fisher (Gibco, USA). Serdemetan was purchased from Topscience (Shanghai, China). RIPA lysate, protease inhibitor, protein phosphatase inhibitor, cell counting Kit-8 (CCK-8), RNase, penicillin/streptomycin and propidium iodide were purchased from Beyotime Biotechnology Company (Shanghai, China). Anti-p53, anti-phospho-p53, anti-cyclin B1, and anti-p21 antibodies were purchased from Abcam (Cambridge, MA, USA). Anti-cyclin D1 antibody were purchased from Cell Signaling Technology (Danvers, MA, USA). Antiphospho-p21 antibody and electro-chemi-luminescence (ECL) kit was purchased from Absin (Shanghai, China). Goat anti-mouse IgG and goat anti-rabbit IgG were purchased from Nachuan biotechnology co., LTD. (Harbin, China).

\section{Cell Culture}

Human glioma U87 and U251 cells were obtained from the cell bank of the Shanghai Biological Institute, Chinese Academy of Science (Shanghai, China). All cells were cultured in high glucose DMEM supplemented with $10 \%$ FBS and $1 \%$ penicillin/streptomycin under $5 \% \mathrm{CO}_{2}$ atmosphere at $37^{\circ} \mathrm{C}$.

\section{Cell Viability Analysis}

The CCK-8 kit was used to analyze the effect of berberine on the viability of U87 and U251 cells. U87 and U251 cells were plated in 96-well plates with $200 \mu \mathrm{L}$ of complete medium in each well. After overnight incubation, the cells adhered to the wells. Media were replaced with cell culture media containing different concentrations of berberine. After 24, 48, and 72 hours of incubation, $10 \mu \mathrm{L}$ of CCK-8 solution was added to each well, and the cells were incubated at $37^{\circ} \mathrm{C}$ for 2 hours. The optical density (OD) of each well was measured at $460 \mathrm{~nm}$.

\section{Clone Formation Experiment}

Tumor cells were plated at a density of $1 \times 10^{3}$ per well in a 6-well plate. The following day, media containing drugs of different concentrations were added to each well. The solution was changed every 3 days. After 2 weeks, the culture was terminated when colonies appeared in the 6-well plate. The supernatant was discarded. Contents were carefully washed three times with phosphatebuffered saline (PBS), fixed with 4\% paraformaldehyde for 30 minutes, stained with $0.1 \%$ crystal violet solution for 20 minutes, carefully rinsed, and dried in air. Colonies of more than 50 cells were quantified under a microscope.

\section{Cell Cycle Analysis}

For cell cycle analysis, U87 and U251 cells were seeded into 6 -well plates at a density of $3 \times 10^{5}$ per well. After the cells adhered to the wells, they were treated with berberine for 24 hours. The cells were collected by digestion with $0.25 \%$ trypsin and fixed in $70 \%$ ethanol at $4^{\circ} \mathrm{C}$ for 24 hours. After resuspension in PBS, Rnase and propidium iodide were added, and contents incubated for $30 \mathrm{~min}$ in the dark. Cell cycle distribution was analyzed by flow cytometry.

\section{Western Blot}

After U87 and U251 cells were treated with berberine for 24 hours, RIPA lysate containing protease inhibitor and protein phosphatase inhibitor was added to extract cellular proteins on ice. An equal amount of protein was separated on a $10 \%$ SDS-PAGE gel and transferred to a polyvinylidene fluoride (PVDF) membrane. The membrane was blocked with TBST containing 5\% skim milk powder at room temperature for 2 hours and incubated with the corresponding diluted primary antibody at $4^{\circ} \mathrm{C}$ overnight. The membrane was subsequently washed three times with TBS-T for 10 minutes and incubated with the corresponding secondary antibody for 1 hour at room temperature. The membrane was washed three times with TBS-T for 10 minutes, and protein content was detected using ECL luminescence.

\section{Lentivirus Transduction}

U251 cells were transduced with wtp53-carrying lentivirus for 24 hours and were screened by adding puromycin. Cells containing the p53 vector expressed green fluorescence when observed under a fluorescence microscope. 


\section{In vivo Tumor Model}

Animal experiments were approved by the Ethics Committee of the First Hospital of Jilin University (Changchun, China) and followed the 5 freedom and 3R principle (Chinese Association for Laboratory Animal Sciences). BALB/c-nu male mice (5-6 weeks of age, weighing 16-18 grams) were purchased from the Charles River Experimental Animal Center (Beijing, China). Mice were housed in a specific pathogen-free environment at a temperature of $25 \pm 2{ }^{\circ} \mathrm{C}$ and humidity of $45 \pm 10 \%$ on a 12 hour:12 hour light/dark cycle with food and water available ad libitum. Tumor cells at a density of $1 \times 10^{6}$ in $200 \mu \mathrm{L}(50 \mu \mathrm{L}$ PBS and $150 \mu \mathrm{L}$ Matrigel) were implanted in each mouse. After approximately 10 days, when the tumor volume reached $180-200 \mathrm{~mm}^{3}$, mice were randomly divided into two groups. The experimental group received daily intraperitoneal injections of berberine $(5 \mathrm{mg} / \mathrm{kg})$ for 2 weeks. The control group received daily intraperitoneal injections with the same volume of solvent. The long diameter (D) and short diameter (d) of the tumor were measured once every two days. Tumor volume (Tv) was calculated by the following formula: $\mathrm{Tv}=0.5 \times \mathrm{D} \times \mathrm{d}^{2}$. On the 14th day after administration, all mice were sacrificed, and tumor tissues were weighed and fixed for subsequent experiments.

\section{Statistical Analyses}

All experiments were repeated independently at least three times, and the experimental data were expressed as mean \pm standard deviation. Statistical analysis was performed using one-way ANOVA. P values $<0.05$ were considered statistically significant.

\section{Results}

\section{Berberine Inhibited Proliferation of Glioma Cells}

Berberine inhibited the proliferation of U87 and U251 cells, and presented the concentration dependence and time dependence of this inhibitory ability (Figure 1B and C). The IC50 values of berberine for U87 cells at 24, 48, and 72 hours were $113.2,62.15$, and $33.07 \mathrm{mg} / \mathrm{L}$, respectively. The IC50 values of berberine for U251 cells at 24,48 , and 72 hours were $149.7,79.5$, and $52.94 \mathrm{mg} / \mathrm{L}$, respectively. Clone formation experiments on U87 and U251 cells demonstrated that berberine inhibited the proliferation of individual tumor cells (Figure 1D).

\section{Berberine Promoted p53 Phosphorylation in U87 Cells and Decreased p53 Content in U25I Cells to Cause Cell Cycle Arrest}

Flow cytometry was used to detect cell cycle changes after treatment of U87 and U251 cells with berberine ( $40 \mathrm{mg} / \mathrm{L})$ for 24 hours (Figure 2A and C). Flowjo software was used to analyze the cell cycle. The number of U87 cells in G1 phase was significantly increased after treatment with berberine. In contrast, the numbers of U251 cells in G1 phase were decreased and $\mathrm{G} 2$ phase were increased, respectively, after treatment with berberine (Figure $2 \mathrm{~B}$ and $\mathrm{D}$ ). In order to observe changes in cell cycle-related proteins, U87 and U251 cells were treated with $0,20,40$, and $80 \mathrm{mg} / \mathrm{L}$ berberine followed by Western blotting. Western blot results revealed that berberine treatment did not significantly change the p53 protein content in U87 cells, while p21 and phosphorylated p53 protein content increased, whereas cyclin D1 protein content decreased. This suggested that berberine caused cell arrest in the G1 phase by promoting p53 protein phosphorylation in U87 cells (Figure 2E). In contrast, berberine treatment induced different effects in U251 cells. p53, p-p53, p21 and cyclin D1 protein content in U251 cells decreased after berberine treatment (Figure 2F).

\section{Interference with Wild-Type p53, Mutant p53 and Their Downstream Molecules can Inhibit the Proliferation of Glioma Cells}

To verify whether cell death could be caused by changes in p53 content, we separately applied Serdemetan (an HDM2 ubiquitin ligase antagonist that acts on p53 wild-type cells to induce early apoptosis and, in cells lacking functional p53, inhibits cell proliferation and delays apoptosis) to U87 and U251 cells. The results showed that cell viability was decreased, as well as that after berberine treatment (Figure 3A and B). Serdemetan and berberine simultaneously increased p-p53 levels in U87 cells (Figure 3C). On the contrary, Serdemetan had no effect on the contents of p53 and p-p53 in U251 cells, but berberine reduced the levels of mutant p53 and p-p53 in U251 cells (Figure 3D).

\section{Berberine Had a More Pronounced Effect on Cell Cycle Arrest in U25I Cells}

\section{Transduced with Wtp53}

To investigate whether berberine inhibited the cell cycle by acting on p53 protein, we transduced U251 cells with 
A
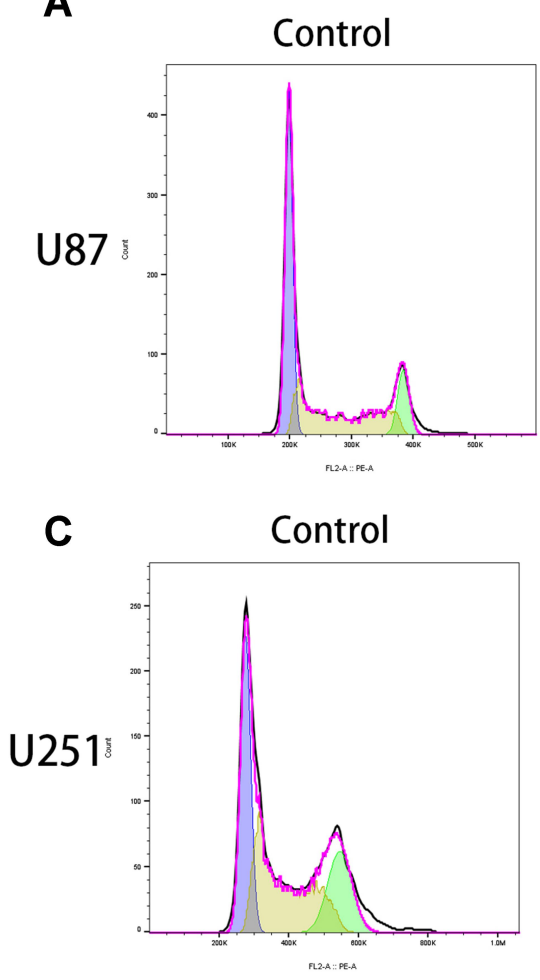

$\mathrm{BBR}(40 \mathrm{mg} / \mathrm{L})$
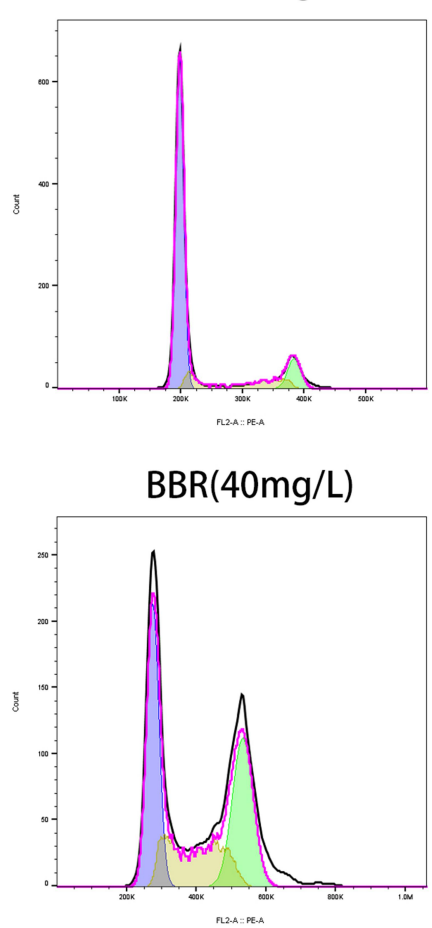

B

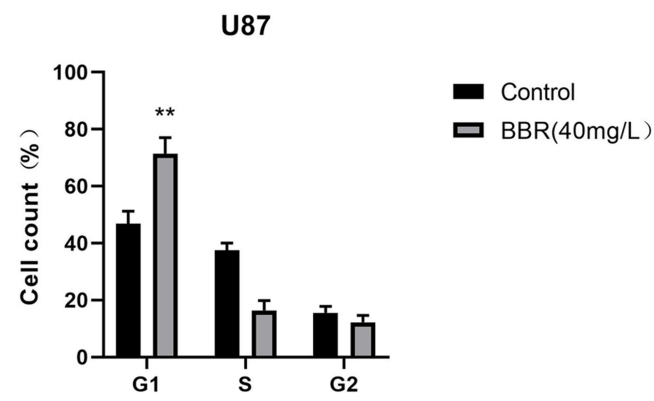

D

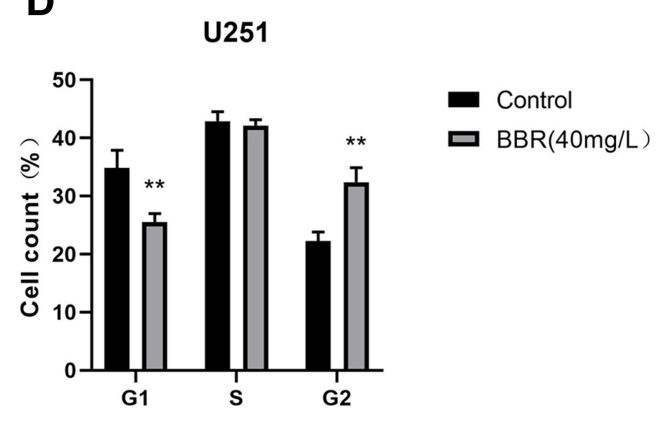

E

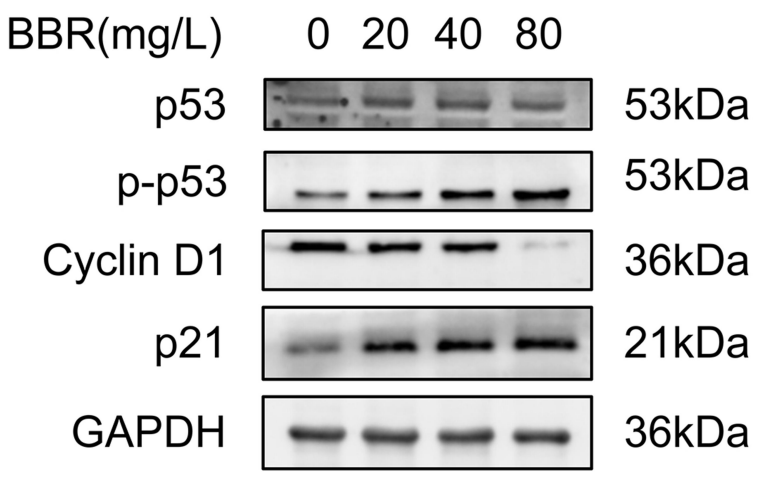

U87
F

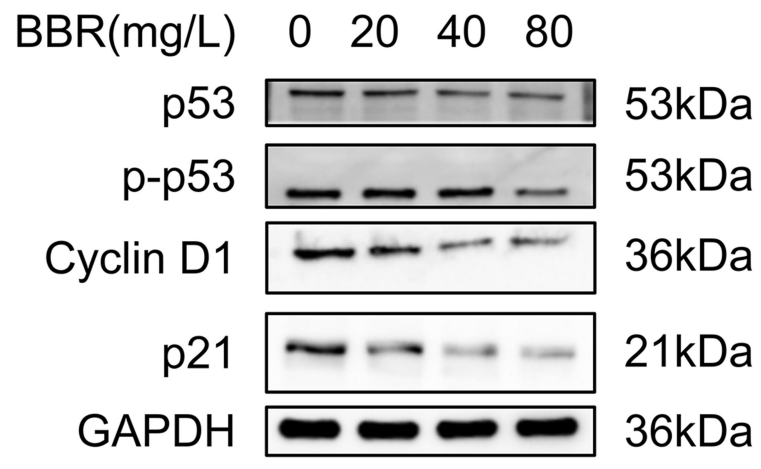

U251

Figure 2 Effect of berberine on the cycle of U87 and U25I cells. (A and B) After U87 cells were treated with berberine (40mg/L) for 24 hours, the number of cells in GI phase increased significantly. (C and D) After U25I cells were treated with berberine (40mg/L) for 24 hours, the number of cells in the GI phase decreased and the number of cells in the G2 phase increased. (E and F) Western blot showed the changes of cycle-related proteins after berberine treatment of U87 and $U 25 \mathrm{I}$ cells for 24 hours. $* *$ indicates BBR $(40 \mathrm{mg} / \mathrm{L})$ treatment group compared with control group, $\mathrm{P}$ values $<0.0 \mathrm{l}$, with statistical significance.

a lentivirus carrying wtp53. Under fluorescence microscopy, U251 cells infected with adenovirus emitted green fluorescence, while those not treated with lentivirus did not emit green fluorescence (Figure 4A). Western blot analysis of lentivirus-transduced cells demonstrated that there was no significant difference in $\mathrm{p} 53$ protein content in the vector group compared with that in normal U251 cells. The p53 protein content in U251 cells transduced with wtp53 lentivirus was significantly increased (Figure 4B and C). Screening with puromycin was performed to obtain a stably transduced cell line, which we named p53-U251 cells. p53-U251 cells were treated with berberine $(40 \mathrm{mg} / \mathrm{L})$ for 24 hours, and the cell cycle was measured by flow cytometry. After transduction with wtp53, the inhibitory effect of berberine on U251 cells was more pronounced. Most cells were in G2 phase 
A

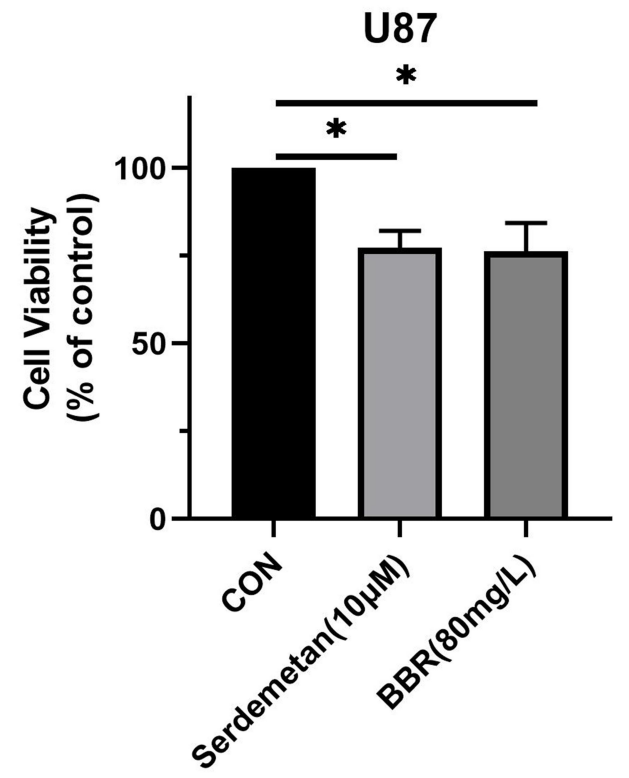

C

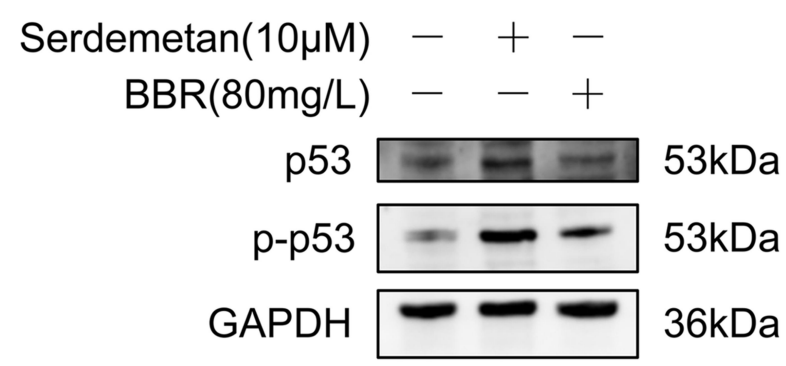

U87
B

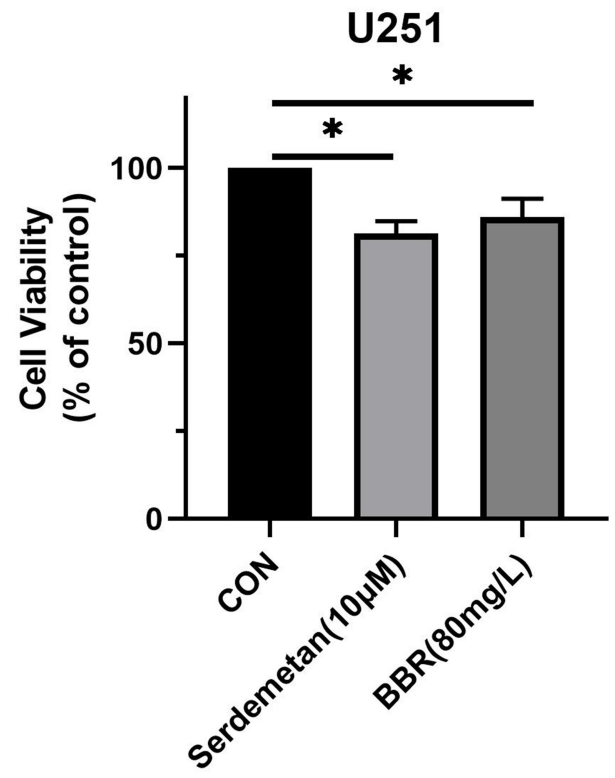

D

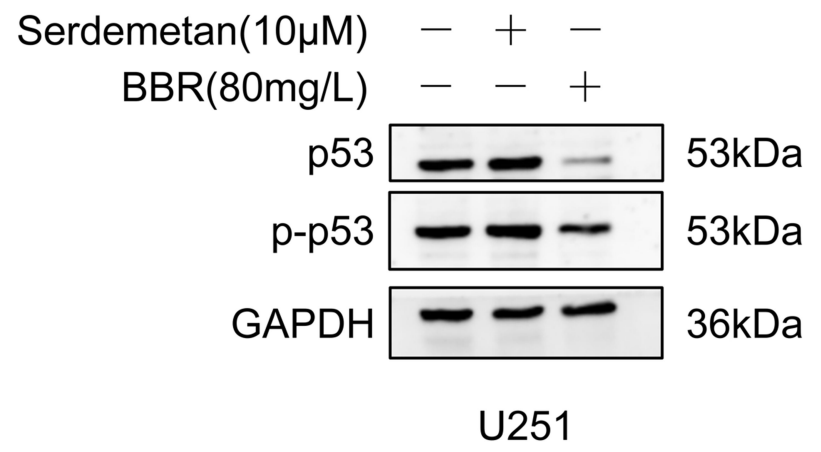

Figure 3 Effects of Serdemetan and berberine on U87 and U25I cells. (A and B) Serdemetan and berberine reduced the cell viability of U87 and U25I cells. (C and D) Western blot showed the effects of Serdemetan and Berberine on the contents of p53 and p-p53 in U87 and U25I cells. * means P<0.05, which is statistically significant.

(Figure 4D and E). Western blot results indicated that cyclin D1 and cyclin B1 protein content in U251 cells and p53-U251 cells was reduced after berberine treatment. After treatment with the same concentration of berberine $(80 \mathrm{mg} / \mathrm{L})$, the protein content in p53-U251 cells decreased to a greater degree. This implied that berberine caused G2 arrest in U251 and p53-U251 cells. In addition, after treatment with the same concentration of berberine $(80 \mathrm{mg} / \mathrm{L}), \mathrm{p} 53$ and phosphorylated p53 protein content in p53-U251 cells was higher than that in U251 cells. No significant change in phosphorylated p21 protein content was observed (Figure 4F-H). To verify the effect of berberine on exogenous p53 content, we used HA-tag to reflect exogenous p53 content in p53 U251 cells. Western blot showed that berberine had no effect on the content of exogenous p53 in p53 U251 cells (Figure 4I).

\section{Berberine Inhibited Glioma Cell Proliferation in vivo}

To verify the antitumor effects of berberine in vivo, U87 and U251 cells were subcutaneously inoculated in nude mice. Approximately 10 days after inoculation when a tumor volume of $180-200 \mathrm{~mm}^{3}$ was attained, mice were randomly divided into either a control group or berberine $(5 \mathrm{mg} / \mathrm{kg})$ treatment group. Tumor volume was smaller (Figure 5A and B), tumor growth rate was slower, and tumor weight was lesser in the berberine-treated group than in the control group (Figure 5C-F). The inhibitory effect of berberine on glioma proliferation was similar in U87 and U251 cell-transplanted tumor tissues. Hematoxylin and eosin staining of tumor tissue in each group revealed that nuclei in the berberine-treated group was lighter in coloration, and cell arrangement was looser (Figure 5G). Immunohistochemical staining and Western 


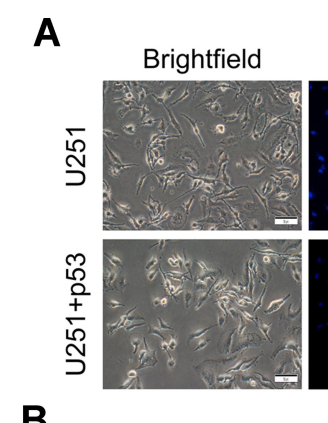

B
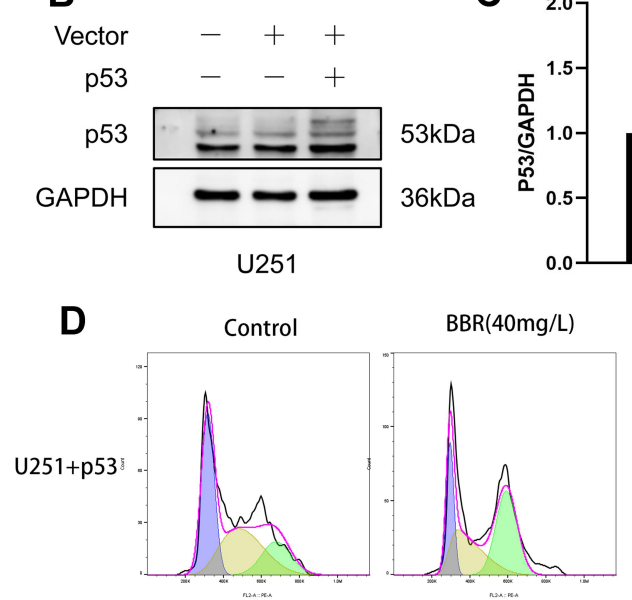

F

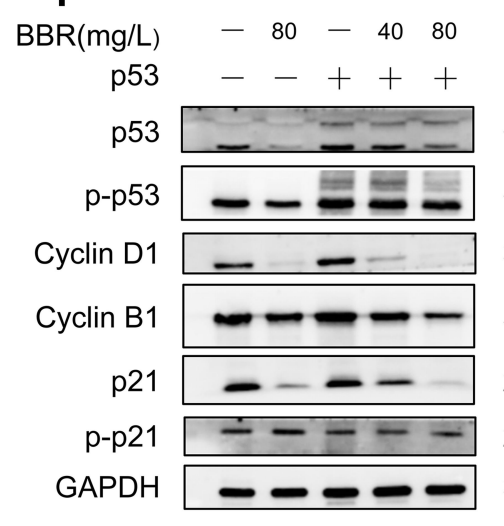

U251

I

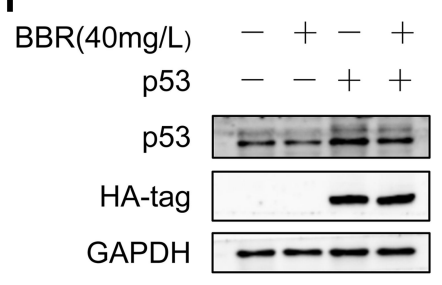

U251

Hoechst

C
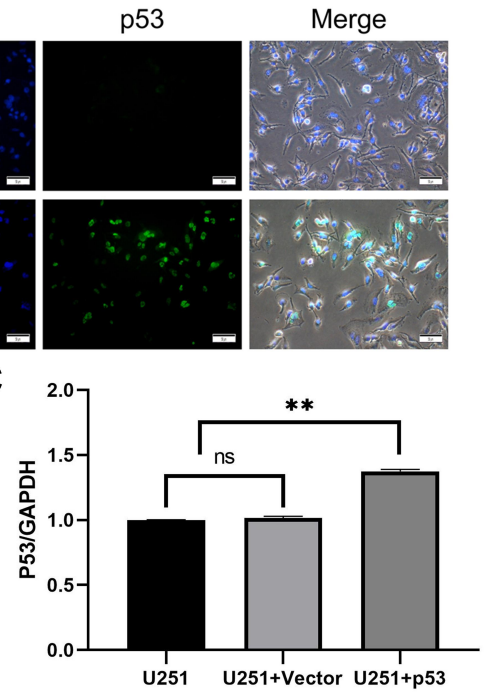

E

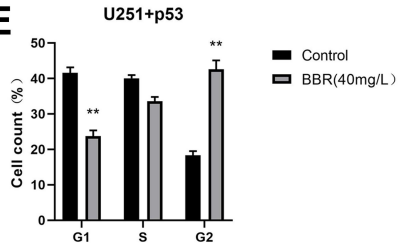

G

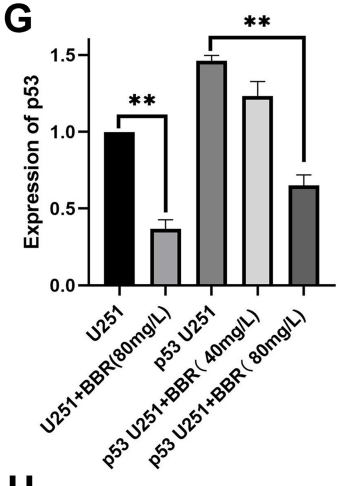

H

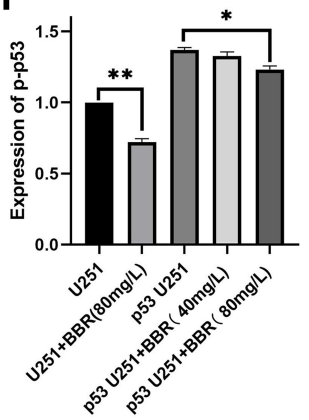

Figure 4 Construction of p53 U25I stably transduced cell line and effect of berberine on the cell cycle of U25I cells transduced with p53. (A) After U25I was transduced with lentivirus, it showed green fluorescence under a fluorescence microscope. U25I cells not transduced with lentivirus did not show green fluorescence. Nuclei were showed in blue. (B) Western blot showed the changes of intracellular $\mathrm{p} 53$ protein after $\mathrm{U} 25 \mathrm{I}$ cells were transduced by lentivirus without and with $\mathrm{P} 53$. GAPDH is used as an internal reference protein. (C) GAPDH protein was used as an internal reference to statistically analyze the expression of p53 protein in three cell lines. (D and E) Flow cytometry showed changes in cell cycle after berberine treated $\mathrm{U} 25 \mathrm{I}$ cells transduced with $\mathrm{p} 53$. (F) Western blots showed changes in cyclin in both cell lines. From left to right, the U25I cell control group, the U25I cell treated group with berberine $(80 \mathrm{mg} / \mathrm{L})$, the $\mathrm{U} 25 \mathrm{I}$ untreated group transduced with $\mathrm{p} 53$, and the $\mathrm{U} 25 \mathrm{I}$ transduced with $\mathrm{p} 53$ treated with berberine ( $40 \mathrm{mg} / \mathrm{L}) \mathrm{Group}$ and $\mathrm{U} 25 \mathrm{I}$ transduced with $\mathrm{p} 53$ were treated with berberine $(80 \mathrm{mg} / \mathrm{L})$. ( $(\mathbf{G}$ and $\mathbf{H})$ Changes of $\mathrm{p} 53$ and $\mathrm{p}-\mathrm{p} 53$ contents after berberine treatment of two cell lines. (I) Western blot showed that berberine had no effect on the content of exogenous $\mathrm{p} 53$ in $\mathrm{p} 53 \mathrm{U} 25 \mathrm{I}$ cells. ns means no statistical significance; * means $\mathrm{P}<0.05$, ** means $\mathrm{P}<0.0 \mathrm{I}$, which is statistically significant. 
A

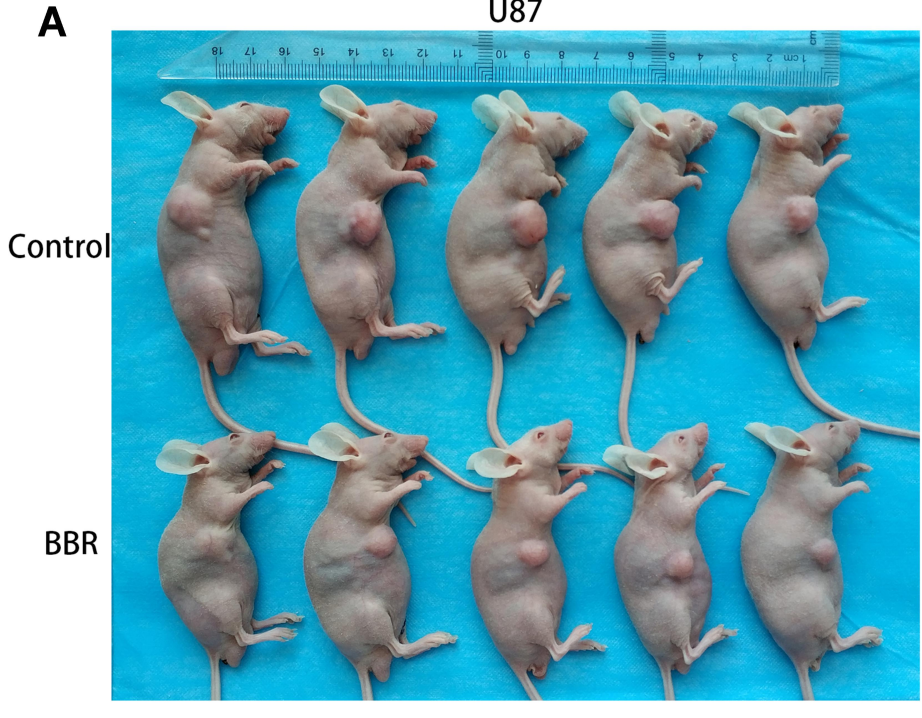

B

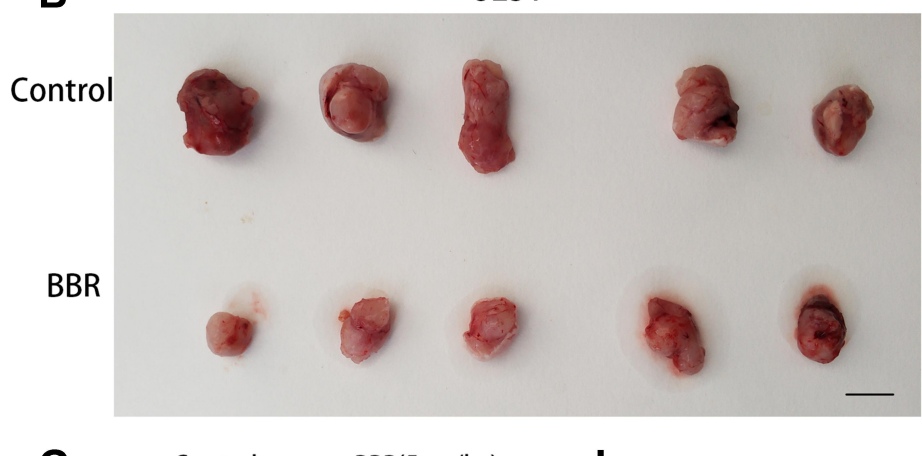

G

U87

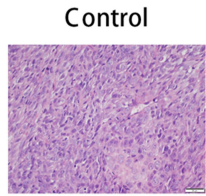

U251

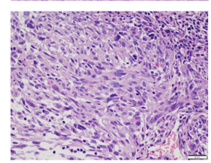

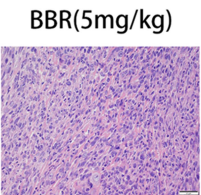

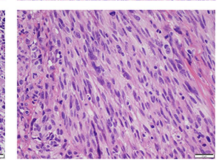

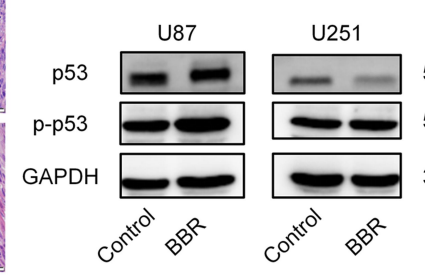

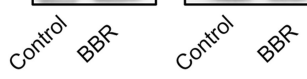

C

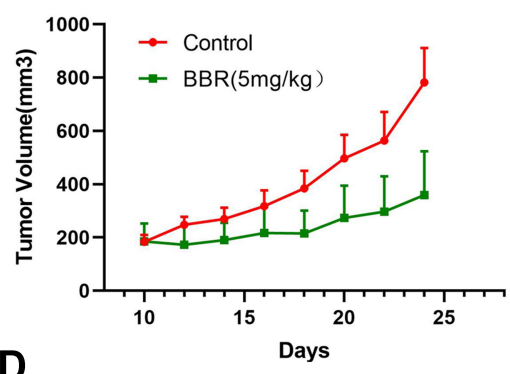

D

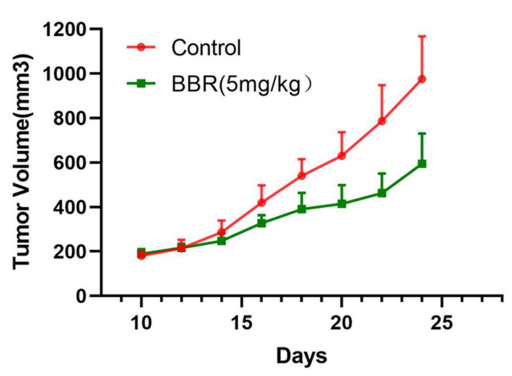

E

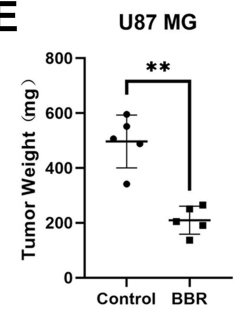

$F$
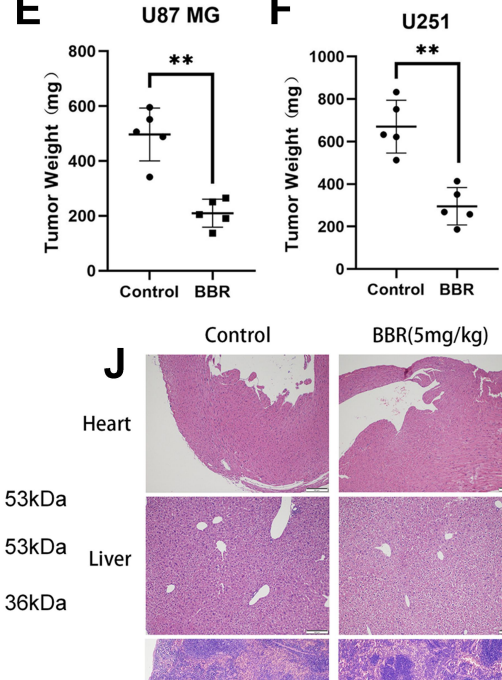

BBR $(5 \mathrm{mg} / \mathrm{kg})$

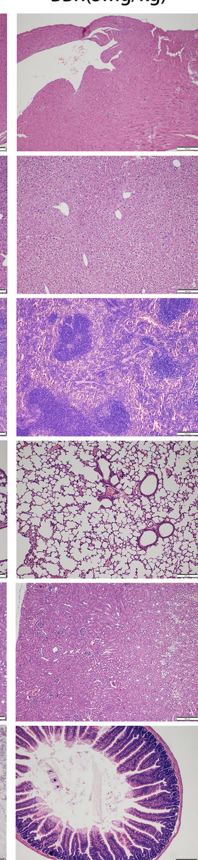

Figure 5 Berberine inhibits glioma growth in vivo. (A and B) After nude mice were inoculated with U87 and U25I cells, the tumors of nude mice were treated with berberine for 14 days, and tumor growth was inhibited. (C and D) The tumor growth curve of the control group and the berberine treatment group, the tumor growth was slow after berberine treatment. (E and $\mathbf{F})$ After 14 days of berberine treatment, the tumor weight in the administration group was smaller. $(\mathbf{G}$ and $\mathbf{H}) \mathrm{HE}$ staining of nude mice transplanted tumor tissue, and immunohistochemical staining with p53 and p-p53 antibodies, the positive cells stained brown. (I) Western blot results of xenograft tumor tissue in nude mice. (J) HE staining of different organs of nude mice in the control and berberine-treated groups. No obvious organic injury was observed in the berberine treatment group under microscope. $* *$ indicates that berberine treatment group $(5 \mathrm{mg} / \mathrm{kg})$ compared with the control group, $\mathrm{P}<0.0 \mathrm{I}$, which has statistical significance. 
blotting results were similar to those of cell experiments (Figure 5H and I). Based on hematoxylin and eosin staining, no obvious organic damage was found after berberine treatment (Figure 5J).

\section{Discussion}

In this study, we examined the inhibitory effects of berberine on human glioma cells. We observed that berberine promoted the phosphorylation of p53 in U87 cells and reduced the content of mutp53 in U251 cells, thereby inhibiting the proliferation of glioma cells.

p53 protein plays a central role in tumor suppression. Due to its short half-life, p53 protein remains very low in cells. ${ }^{23}$ Under stress conditions such as hypoxia and DNA damage, p53 protein is activated by phosphorylation, acetylation, and methylation. Its content in the cell rapidly rises to a high level, and it subsequently enters the nucleus where it regulates downstream gene transcription and participates in the cell cycle, apoptosis, drug resistance, autophagy, proliferation, and other processes. ${ }^{24}$ TP53 is the most frequently mutated gene in human cancers. Indeed, mutations in TP53 are present in more than half of all cancers. ${ }^{11,12}$ p53 mutations are often missense mutations (replacement of a single amino acid), and most mutations occur in the DNA binding region (DBD) of p53 which binds to target genes, enabling p53 to act as a transcription factor. ${ }^{25}$ Among the p53 mutation sites, six codons are considered to be hotspot mutants: R175, G245, R248, R249, R273, and R282. More than 25\% of the mutations occur at these sites. ${ }^{26}$ In U251 glioma cells, p53 has a mutation at the R273 site. ${ }^{27}$ At this site, histidine has replaced arginine, conferring p53 a tumor-enhancing effect.

p53 mutations cause loss of the tumor-suppressing functions of wtp53, negatively regulate the activity of wtp53, and play a tumor-promoting role. ${ }^{25,28}$ To date, many mutp53 gain-of-function (GOF) effects have been identified, including promoting tumor cell migration, invasion, drug resistance, proliferation, and hypoxia resistance, destroying appropriate tissue structures, and promoting cancer metabolism. ${ }^{29}$ As mutp53 cannot be degraded by normal pathways, it often accumulates to high levels in tumor cells. ${ }^{30}$ Notably, the accumulation of mutp53 is key to its GOF effects. ${ }^{31}$ Further, mutp53 tends to be highly expressed in tumor tissues and is scarcely present in normal tissues. ${ }^{32}$ Therefore, therapeutic strategies targeting mutp53 may be the key to treating p53 mutant cancers. The current treatment methods for mutp53 are mainly divided into two categories: (1) promoting the degradation of mutp53; (2) promoting the functional recovery of mutp53. ${ }^{33}$ At present, the main drugs that promote the degradation of mutp53 are geldanamycin, 17AAG, ganetespib, histone, and gambogic acid. Representative drugs that promote the recovery of mutp53 function are PRIMA-1 and ZMC-1. ${ }^{23}$

Glioma is the most common malignant tumor in the skull and has poor prognosis due to its excessive growth, diffuse invasion, and resistance to chemotherapy. ${ }^{34,35}$ To date, temozolomide is the main drug used for clinical treatment of gliomas, but chemotherapy resistance remains an issue. ${ }^{8}$ Many anti-glioma drugs currently studied are often unsuitable for clinical application as they are unable to penetrate the blood-brain barrier. Berberine is an isoquinoline alkaloid found in natural plants. Berberine has antiinflammatory, blood-sugar-lowering, anti-microbial, and anti-tumor effects. ${ }^{19,21}$ Berberine has been used for many years as a non-prescription drug for the treatment of bacterial diarrhea in China ${ }^{36}$. Berberine is also considered a potential drug for the treatment of hyperlipidemia and diabetes, which has been proved in many clinical trials. ${ }^{37}$ All these prove that berberine has high safety. Notably, berberine can pass through the blood-brain barrier to exert its anti-tumor effects. ${ }^{20,38}$ Our previous studies have demonstrated that berberine has a destructive effect on a variety of glioma cells, including T98G, LN18, C6, SHG44, and LN229 cells, and its main mechanism is the induction of glioma cells programmed cell death through ERK1/2-mediated mitochondrial damage. ${ }^{20}$ In addition, berberine has been proved to regulate autophagy, apoptosis, invasion and chemotherapy sensitization of glioma cells in various ways. ${ }^{21}$ In this study, we tested the inhibitory effects of berberine on U87 and U251 cells. Our results indicated that berberine inhibited the proliferation of glioma cells, and this inhibitory ability presented the concentration dependence and time dependence. We observed that in U87 cells, berberine promoted the phosphorylation of wtp53 Total p53 content was not significantly changed, thereby promoting downstream $\mathrm{p} 21$ protein transcription, reducing cyclin D1 protein content, and preventing U87 cells from progressing to $\mathrm{S}$ phase from G1, causing G1 arrest, and inhibition of U87 cell proliferation. In U251 cells, as the structure of mutp53 protein DNA (p53 R273H) is altered in its DBD, tumor suppressor functions of wtp53 are lost. Berberine treatment resulted in a decrease in the content of mutp53, p21, and cyclin D1 protein in U251 cells. We speculate that in U251 cells, mutp53 was still able to induce p21 transcription, increase the content of cyclin D1, and promote progression into the $\mathrm{S}$ phase; these effects were perturbed by berberine. However, after treatment of U251 
cells with Serdemetan, the content of mutant p53 was not changed, but the cell viability was decreased. This may be due to the fact that Serdemetan acts on the p53 mutant cells, accelerates the protease-regulated p21 degradation and counteracts the transcription induction of $\mathrm{p} 21$ by mutp53, thus inducing the S-phase delay and leading to the preferential apoptosis of S-phase cells. ${ }^{39}$ To further investigate the interaction between p53 and berberine, we expressed wtp53 gene in U251 cells. Following berberine treatment, we observed an attenuation in the increased content of phosphorylated p53 compared with normal U251 treated with berberine, as berberine may have promoted the phosphorylation of wtp53. Cyclin D1 and cyclin B1 content decreased to a greater extent, indicating that the inhibitory effect of berberine was more pronounced after transduction of wtp53. In cells containing both wild-type and mutant $\mathrm{p} 53$, berberine facilitates both promotes the phosphorylation of wtp53 but also reduces mutp53 content, resulting in an additive effect. Immunohistochemistry of U251 xenograft tissue revealed mutp53 expression in tumor tissue, suggesting that mutp53 tends to accumulate in tumors and promote tumor cell GOF. In summary, our study suggests that berberine promotes the phosphorylation of wtp53 and reduces the content of mutp53, thereby causing cell cycle arrest and inhibition of U87 and U251 cell proliferation (Figure 6).

The heterogeneity of gliomas confers malignant features and a poor clinical prognosis, with $\mathrm{p} 53$ protein mutations exist in more than $50 \%$ of glioma. The accumulation of mutant $\mathrm{p} 53$ is considered to be the key to its GOF, and promoting the degradation of mutant p53 is considered to be an effective means of treating p53 mutant tumors. Berberine can promote the phosphorylation of wild-type p53 and reduce the content of mutant $\mathrm{p} 53$. Therefore, berberine can be used as a potential drug to treat wild-type and mutant p53 glioma.

However, we did not verify the molecular mechanism of mutp53, p21 and cyclin D1 in U251 cells, especially the same changes in the content of p21 and cyclin D1 after berberine treatment. We speculate that mutp53 may directly promote the transcription of cyclin D1 to cause such changes, which we will verify in future experiments.

\section{Conclusion}

In conclusion, we have demonstrated in this study that berberine promoted the phosphorylation of p53 in U87 cells and reduced the content of mutp53 in U251 cells.

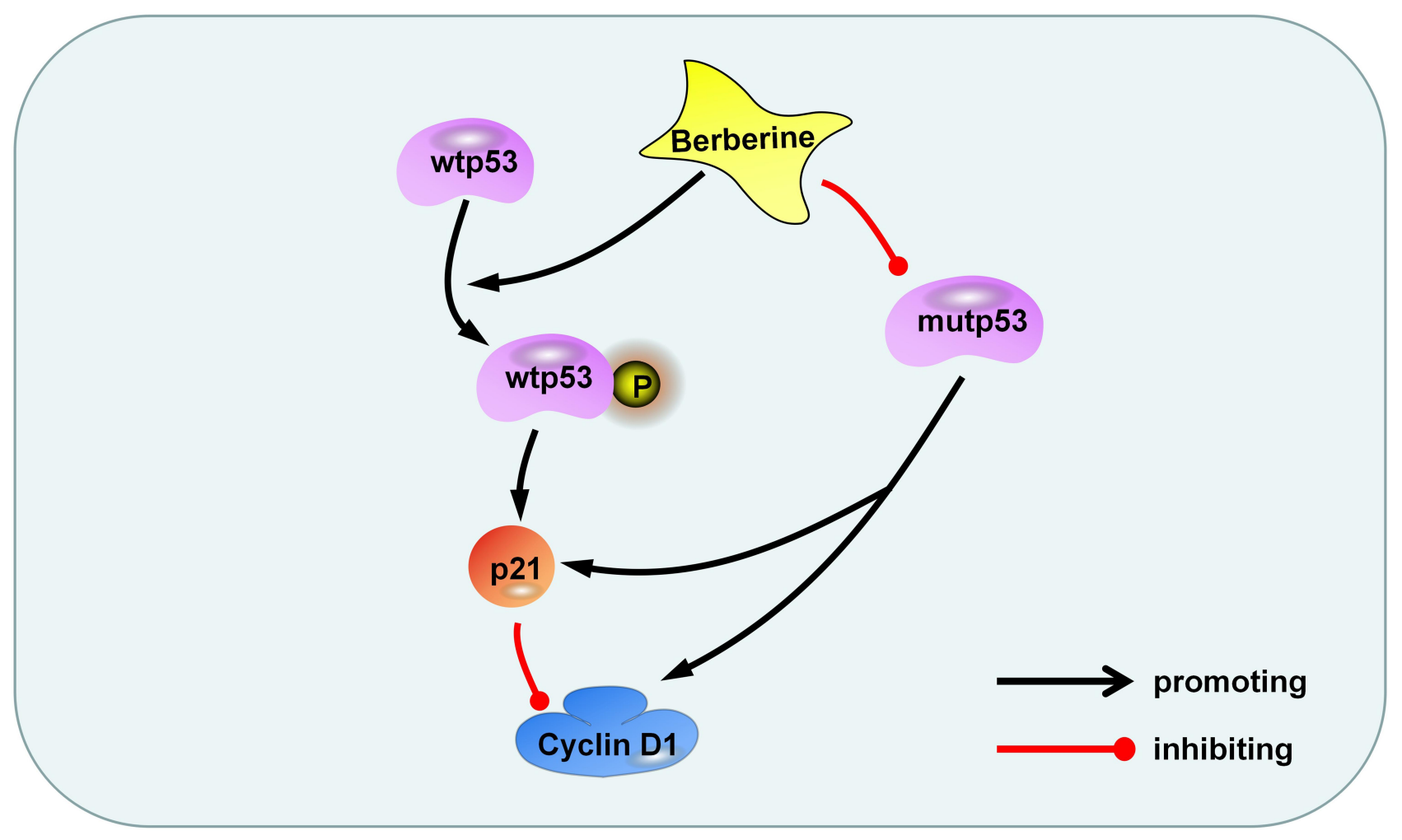

Figure 6 Berberine inhibits glioma cell proliferation through the p53 pathway. Berberine promotes the phosphorylation of wtp53, activates p2I, and inhibits cyclin DI protein. On the other hand, berberis inhibits mutp53 and reduces the content of $\mathrm{p} 2 \mathrm{I}$ and cyclin DI. 
Berberine inhibit glioma cell proliferation via the $\mathrm{p} 53 /$ p21/cyclin D1 pathway and thus, may be a potential p53 targeting therapeutic for glioma. It is worth noting that we do not know how berberine promotes the degradation of mutp53, and we will continue to explore the molecular mechanism of berberine and mutp53 in future studies.

\section{Acknowledgments}

This work was supported by Scientific Research Foundation of Jilin Province (20180101158JC, 20200201613JC), Research and Planning Project of the 13th Five-Year Science and Technology Project of Jilin Provincial Department of Education (JJKH20180191KJ) and Interdisciplinary Innovation Project of First Hospital of Jilin University (JDYYJC001). We thank the Neurosurgery Laboratory of the First Hospital of Jilin University for providing the laboratory equipment.

\section{Disclosure}

The authors declare that they have no conflicts of interest.

\section{References}

1. Ostrom QT, Bauchet L, Davis FG, et al. The epidemiology of glioma in adults: a "state of the science" review. Neuro Oncol. 2014;16 (7):896-913. doi:10.1093/neuonc/nou087

2. Meng X, Duan C, Pang H, et al. DNA damage repair alterations modulate M2 polarization of microglia to remodel the tumor microenvironment via the p53-mediated MDK expression in glioma. EBioMedicine. 2019;41:185-199. doi:10.1016/j. ebiom.2019.01.067

3. Cai J, Zhang J, Wu P, et al. Blocking LINC00152 suppresses glioblastoma malignancy by impairing mesenchymal phenotype through the miR-612/AKT2/NF-kappaB pathway. $J$ Neurooncol. 2018;140:225-236. doi:10.1007/s11060-018-2951-0

4. Reifenberger G, Wirsching HG, Knobbe-Thomsen CB, Weller M. Advances in the molecular genetics of gliomas - implications for classification and therapy. Nat Rev Clin Oncol. 2017;14:434-452.

5. Gilbert MR, Dignam JJ, Armstrong TS, et al. A randomized trial of bevacizumab for newly diagnosed glioblastoma. $N$ Engl $J$ Med. 2014;370:699-708. doi:10.1056/NEJMoa1308573

6. Li Y, Zhang MC, Xu XK, et al. Functional diversity of p53 in human and wild animals. Front Endocrinol (Lausanne). 2019;10:152. doi:10.3389/fendo.2019.00152

7. Stepinski D. Nucleolus-derived mediators in oncogenic stress response and activation of p53-dependent pathways. Histochem Cell Biol. 2016;146:119-139.

8. Wang N, Zhang Q, Luo L, Ning B, Fang Y. beta-asarone inhibited cell growth and promoted autophagy via P53/Bcl-2/Bclin-1 and P53/ AMPK/mTOR pathways in human glioma U251 cells. $J$ Cell Physiol. 2018;233(3):2434-2443. doi:10.1002/jcp.26118

9. Vousden $\mathrm{KH}$, Prives $\mathrm{C}$. Blinded by the light: the growing complexity of p53. Cell. 2009;137(3):413-431. doi:10.1016/j.cell.2009.04.037

10. Muller PA, Vousden KH. p53 mutations in cancer. Nat Cell Biol. 2013;15:2-8. doi:10.1038/ncb2641
11. Muller PA, Vousden KH. Mutant p53 in cancer: new functions and therapeutic opportunities. Cancer Cell. 2014;25(3):304-317. doi:10.1016/j.ccr.2014.01.021

12. Freed-Pastor WA, Prives C. Mutant p53: one name, many proteins. Genes Dev. 2012;26(12):1268-1286. doi:10.1101/gad.190678.112

13. Yang HW, Chung M, Kudo T, Meyer T. Competing memories of mitogen and p53 signalling control cell-cycle entry. Nature. 2017;549 (7672):404-408. doi:10.1038/nature23880

14. Chen YJ, Lin JK, Lin-Shiau SY. Proliferation arrest and induction of CDK inhibitors p21 and p27 by depleting the calcium store in cultured C6 glioma cells. Eur J Cell Biol. 1999;78(11):824-831. doi:10.1016/S0171-9335(99)80033-8

15. Li M, Li L, Zhang L, et al. 1, 25-Dihydroxyvitamin D3 suppresses gastric cancer cell growth through VDR- and mutant p53-mediated induction of p21. Life Sci. 2017;179:88-97. doi:10.1016/j. Ifs.2017.04.021

16. Morton JP, Timpson P, Karim SA, et al. Mutant p53 drives metastasis and overcomes growth arrest/senescence in pancreatic cancer. Proc Natl Acad Sci U $\quad S$ A. 2010;107:246-251. doi:10.1073/ pnas.0908428107

17. Ruan H, Zhan YY, Hou J, et al. Berberine binds RXRalpha to suppress beta-catenin signaling in colon cancer cells. Oncogene. 2017;36(50):6906-6918. doi:10.1038/onc.2017.296

18. Zou K, Li Z, Zhang Y, et al. Advances in the study of berberine and its derivatives: a focus on anti-inflammatory and anti-tumor effects in the digestive system. Acta Pharmacol Sin. 2017;38(2):157-167. doi:10.1038/aps.2016.125

19. Ma W, Zhu M, Zhang D, et al. Berberine inhibits the proliferation and migration of breast cancer ZR-75-30 cells by targeting Ephrin-B2. Phytomedicine. 2017;25:45-51. doi:10.1016/j. phymed.2016.12.013

20. Sun Y, Yu J, Liu X, et al. Oncosis-like cell death is induced by berberine through ERK1/2-mediated impairment of mitochondrial aerobic respiration in gliomas. Biomed Pharmacother. 2018;102:699-710. doi:10.1016/j.biopha.2018.03.132

21. Asemi Z, Behnam M, Pourattar MA, Mirzaei H, Razavi ZS, Tamtaji OR. Therapeutic potential of berberine in the treatment of glioma: insights into its regulatory mechanisms. Cell Mol Neurobiol. 2020. doi:10.1007/s10571-020-00903-5

22. Jabbarzadeh Kaboli P, Rahmat A, Ismail P, Ling KH. Targets and mechanisms of berberine, a natural drug with potential to treat cancer with special focus on breast cancer. Eur $J$ Pharmacol. 2014;740:584-595. doi:10.1016/j.ejphar.2014.06.025

23. Yue X, Zhao Y, Xu Y, Zheng M, Feng Z, Hu W. Mutant p53 in cancer: accumulation, gain-of-function, and therapy. $J$ Mol Biol. 2017;429:1595-1606. doi:10.1016/j.jmb.2017.03.030

24. Levine AJ, Hu W, Feng Z. The P53 pathway: what questions remain to be explored? Cell Death Differ. 2006;13:1027-1036. doi:10.1038/ sj.cdd. 4401910

25. Brosh R, Rotter V. When mutants gain new powers: news from the mutant p53 field. Nat Rev Cancer. 2009;9:701-713. doi:10.1038/ nrc2693

26. Acin S, Li Z, Mejia O, Roop DR, El-Naggar AK, Caulin C. Gain-offunction mutant $\mathrm{p} 53$ but not $\mathrm{p} 53$ deletion promotes head and neck cancer progression in response to oncogenic K-ras. J Pathol. 2011;225:479-489. doi:10.1002/path.2971

27. Brazdova M, Quante T, Togel L, et al. Modulation of gene expression in U251 glioblastoma cells by binding of mutant p53 R273H to intronic and intergenic sequences. Nucleic Acids Res. 2009;37:1486-1500. doi:10.1093/nar/gkn1085

28. Meek DW. Regulation of the p53 response and its relationship to cancer. Biochem J. 2015;469:325-346.

29. Muller PA, Caswell PT, Doyle B, et al. Mutant p53 drives invasion by promoting integrin recycling. Cell. 2009;139:1327-1341. doi:10.1016/j.cell.2009.11.026 
30. Liu J, Zhang C, Hu W, Feng Z. Tumor suppressor p53 and its mutants in cancer metabolism. Cancer Lett. 2015;356:197-203. doi:10.1016/j. canlet.2013.12.025

31. Oren M, Rotter V. Mutant p53 gain-of-function in cancer. Cold Spring Harb Perspect Biol. 2010;2:a001107. doi:10.1101/cshperspect.a001107

32. Alexandrova EM, Yallowitz AR, Li D, et al. Improving survival by exploiting tumour dependence on stabilized mutant p53 for treatment. Nature. 2015;523(7560):352-356. doi:10.1038/nature14430

33. Bykov VJN, Eriksson SE, Bianchi J, Wiman KG. Targeting mutant p53 for efficient cancer therapy. Nat Rev Cancer. 2018;18(2):89-102. doi:10.1038/nrc.2017.109

34. Xiao M, An Y, Wang F, et al. A chimeric protein PTEN-L-p53 enters U251 cells to repress proliferation and invasion. Exp Cell Res. 2018;369(2):234-242. doi:10.1016/j.yexcr.2018.05.023

35. Han B, Meng X, Chen H, et al. Epigenetic silencing of miR-338 facilitates glioblastoma progression by de-repressing the pyruvate kinase M2-beta-catenin axis. Aging (Albany NY). 2017;9 (8):1885-1897. doi:10.18632/aging.101271
36. Kumar A, Ekavali CK, Mukherjee M, Pottabathini R, Dhull DK. Current knowledge and pharmacological profile of berberine: an update. Eur J Pharmacol. 2015;761:288-297. doi:10.1016/j. ejphar.2015.05.068

37. Cicero AF, Baggioni A. Berberine and its role in chronic disease. $A d v$ Exp Med Biol. 2016;928:27-45.

38. Wang S, He B, Hang W, et al. Berberine alleviates tau hyperphosphorylation and axonopathy-associated with diabetic encephalopathy via restoring PI3K/Akt/GSK3beta pathway. J Alzheimers Dis. 2018;65:1385-1400. doi:10.3233/JAD-180497

39. Thompson T, Andreeff M, Studzinski GP, Vassilev LT. 1,25dihydroxyvitamin D3 enhances the apoptotic activity of MDM2 antagonist nutlin-3a in acute myeloid leukemia cells expressing wild-type p53. Mol Cancer Ther. 2010;9:1158-1168. doi:10.1158/ 1535-7163.MCT-09-1036

\section{Publish your work in this journal}

OncoTargets and Therapy is an international, peer-reviewed, open access journal focusing on the pathological basis of all cancers, potential targets for therapy and treatment protocols employed to improve the management of cancer patients. The journal also focuses on the impact of management programs and new therapeutic agents and protocols on patient perspectives such as quality of life, adherence and satisfaction. The manuscript management system is completely online and includes a very quick and fair peer-review system, which is all easy to use. Visit http://www.dovepress.com/ testimonials.php to read real quotes from published authors. 